\title{
Advanced Vascular Access in Small Animal Emergency and Critical Care
}

\author{
Jack A. Lee*, Liz-Valéry S. Guieu, Geneviève Bussières and Christopher K. Smith \\ Small Animal Clinical Sciences, College of Veterinary Medicine, University of Tennessee, Knoxville, TN, United States
}

In canine and feline patients presenting in a state of hemodynamic collapse, obtaining vascular access can be challenging. Delays in achieving vascular access interfere with delivery of patient care. In human medicine, definitions of difficult vascular access are variable and include the need for multiple placement attempts or involvement of specialized teams and equipment. Incidence and risk factors for difficult vascular access have not been well studied in veterinary patients, which limits understanding of how best to address this issue. Alternatives to percutaneous peripheral or central intravenous catheterization in dogs and cats include venous cutdowns, umbilical access in newborns, corpus cavernosum access in males, ultrasound-guided catheterization, and intraosseous catheterization. In recent years, advances in ultrasonography and

OPEN ACCESS

Edited by:

Charlotte Sandersen University of Liège, Belgium

Reviewed by:

Thomas H. Edwards, United States Army Institute of Surgical Research, United States Jennifer Devey, Fox Valley Animal Referral Center (FVARC), United States

*Correspondence:

Jack $A$. Lee

jlee184@vols.utk.edu

Specialty section:

This article was submitted to Veterinary Emergency and Critical

Care Medicine

a section of the journal

Frontiers in Veterinary Science

Received: 30 April 2021 Accepted: 08 November 2021 Published: 29 November 2021

Citation:

Lee JA, Guieu L-VS, Bussières G and Smith CK (2021) Advanced Vascular Access in Small Animal Emergency and Critical Care.

Front. Vet. Sci. 8:703595. doi: 10.3389/fvets.2021.703595 intraosseous access techniques have made these more accessible to veterinary practitioners. These vascular access techniques are reviewed here, along with advantages, limitations, and areas for future study of each technique.

Keywords: difficult vascular access, intraosseous, cutdown, emergency, veterinary, ultrasound

\section{INTRODUCTION}

Multiple definitions of difficult vascular access (DVA) exist in the human medical literature, which encompass the need for multiple placement attempts, specialized equipment, and highly experienced teams (1-3). In human pediatrics, $\sim 50 \%$ of patients are successfully catheterized on the first attempt; $5-33 \%$ of patients require more than two placement attempts; and in one study, $5 \%$ could not have a peripheral intravenous (IV) catheter placed at all $(4,5)$. Children below 2 years old are the most challenging and time-consuming to catheterize, which is thought to be due to their smaller size, difficulty in palpating vasculature, and possibly patient non-compliance $(1,3,5)$. In pediatrics, scoring systems such as the difficult IV access (DIVA) scoring tool based on age, vein visualization, and vein palpation have been developed and may be helpful in identifying patients at high risk for DVA $(5,6)$. A similar scoring system has also been developed for adults based on vessel visibility, palpability, and size, as well as a history of DVA and emergency indication for surgery $(7,8)$. Besides age, factors that may contribute to DVA in humans include dehydration, hypotension, metabolic disease such as diabetes mellitus, cardiovascular disease, scarring from recurrent catheterization, skin lesions associated with trauma or burn injury, and obesity (2, 9-11). The consequences of DVA can be severe, including discomfort associated with repeated attempts and delays in necessary treatments, which can be life-threatening (12-14). In human medicine, use of dedicated vascular access teams for selection, placement, and maintenance of vascular access devices helps to improve success rates and decreases complications $(2,15)$. These teams combine dedicated nurses, technicians, and doctors with formal training; teams may subspecialize in specific procedures (e.g., peripheral catheters and central venous catheters). Recently, the development of smartphone-based applications has also been investigated to help with vein identification based 
on multispectral Wiener estimation using the phone camera (16, 17). These appear to be a helpful point-of-care way to improve vein visualization but have not been clinically investigated. Other phone applications have been developed to aid in appropriate vascular access device selection in individual patients (18). To the authors' knowledge, these have not been evaluated for use in veterinary patients.

A consensus definition of DVA does not exist in veterinary medicine, and its prevalence in canine and feline patients has not been well-reported, particularly in critically ill patients. Successful first-time peripheral catheter placement rates in dogs and cats have been documented in $51-94 \%$ of attempts in a general patient population $(19,20)$, with individual experience being an important factor for success (19). In the critically ill veterinary patient, the incidence of DVA can be expected to increase given the multitude of presenting comorbidities such as hypotension, vasoconstriction, trauma to the desired access sites, and non-compliance.

Gaining venous access is vital to patient care and allows for blood sampling and delivery of therapies such as medications, fluids, and blood products. The most common initial approach is typically peripheral IV catheterization, while central catheterization or other advanced vascular access options may be considered in certain patients. In general, short, smalldiameter catheters decrease the risk of thrombophlebitis (21-23). Short, large-gauge catheters allow for more rapid administration of fluids (24). With longer catheters, rapid delivery of fluids can be challenging due to the proportional increase in resistance that accompanies length, as described by the Hagen-Poiseuille equation. This is particularly true in smaller-bore catheters $(24,25)$. A number of alternatives to traditional peripheral and central IV catheterization have become available to small animal practitioners in recent years and are described here.

\section{VENOUS CUTDOWN}

In cases when visualization or palpation of the vessel is challenging, peripheral and central catheter placement can be facilitated with a cutdown. This can be achieved with readily available supplies, though depending on operator skill can be more time-consuming than some alternatives. The cephalic, lateral saphenous, or jugular veins are common sites for cutdown approaches in dogs. In dogs with large ears, such as Basset Hounds and Dachshunds, the auricular vein approach can also be used. In cats, the medial saphenous approach can also be used $(26,27)$. The technique is described elsewhere $(26,27)$. Contraindications include trauma to the desired placement area or anywhere along the limb proximal to the insertion site along the course of the desired vessel. Infection at the desired site is an additional contraindication. Coagulopathy is a relative contraindication, particularly for larger vessels where the risk of life-threatening hemorrhage should be considered and other techniques pursued if possible (28). Complications may

Abbreviations: BUN, blood urea nitrogen; DVA, difficult vascular access; IO, intraosseous; IV, intravenous; PCV, packed cell volume; TP, total protein; US, ultrasound; UV, umbilical vein. include vessel damage, hemorrhage, hematoma, local infection, thrombosis, and nerve injury $(26,27)$. Cutdowns may have an increased risk of infection and hemorrhage compared with conventional catheter placement (20-23). The frequency of complications in humans has been reported to be between 2 and $15 \%$, though recent data are lacking $(28,29)$. Complications are minimized by removal of the catheter as soon as another option is available $(24,29)$. The average time to perform the technique in humans has been reported to be between 5.6 and $7.5 \mathrm{~min}(30,31)$. Successful peripheral IV catheter placement with a cutdown was reported as $69 \%$ in a cadaver study and $85 \%$ in a hypotensive population $(29,31)$. In humans, use of the Seldinger technique and other advanced vascular access options have made cutdowns less popular (28). Use of cutdowns in veterinary medicine has been sparsely documented. To the authors' knowledge, cutdowns have not been clinically studied in veterinary patients, nor has the rate of complications been reported. Further investigation is needed to compare outcomes with other available options in veterinary patients.

As an alternative to full cutdown, a technique described as facilitative incision or relief hole can be utilized for severely dehydrated patients, or those with thick skin, to reduce the tension and friction of the skin against the catheter (27). A skin incision of about $1-2 \mathrm{~mm}$ is made directly over the vessel extending through the dermis using a number 11 blade or appropriately sized hypodermic needle with or without local anesthesia (rarely needed). Care should be taken to avoid the vessel when making the relief incision. This technique, if successful, has less chance of infection or healing issues but does not allow for visualization of the vein (27).

\section{CORPUS CAVERNOSUM}

An infrequently utilized alternative to peripheral venous catheterization for male patients with DVA is catheterization of the corpus cavernosum. This technique is effective due to the substantial venous drainage from this structure and requires no specialized equipment. After aseptic preparation, access is achieved using a $19-\mathrm{G}$ catheter inserted at an oblique angle toward the radix through the skin and into the corpus cavernosum (located on the lateral aspect of the penis, caudal to the os penis) (32-34). Placement is confirmed via aspiration of blood. One known limitation would be in pelvic or penile trauma, where venous return may be impaired. Human literature on resuscitation using this technique is limited (35), though the treatment of erectile dysfunction using injections into the corpus cavernosum suggests that while the potential for complications such as fibrosis and penile dysfunction are possible, they are rare (34). In canine experimental models, placement of a catheter into the corpus cavernosum achieved sufficient flow rates to resuscitate experimentally induced hypovolemic animals, as well as to administer epinephrine and atropine $(33,36)$. Administration of blood products and phenobarbital has also been successful (32-34). Administration of other emergency 
medications has not been studied, and further clinical work is required to fully assess the incidence of complication and limitations of this modality.

\section{ULTRASOUND GUIDANCE}

Ultrasound (US) guidance has been incorporated in both peripheral and central venous access procedures. High-quality US is becoming increasingly available in veterinary medicine. Daily point-of-care use in the emergency room (ER) and intensive care unit (ICU) is increasing, and the cost of this equipment is decreasing. Ultrasonography provides practitioners with the ability to visualize the vessel for catheterization. This can be particularly helpful when palpation or visualization of vascular landmarks is difficult. It can also allow for identification of any abnormal surrounding anatomy. High-frequency linear probes $(>4 \mathrm{MHz}$ ) are most commonly used for this purpose, though convex probes have been used in small patients. This technique has been well-described in people and is described here in Figure 1A (37-39). To maintain sterility, the probe is placed within a sterile sheath or sterile glove containing US gel (40-42). Application of alcohol to the intended site can be used to improve visualization. Vessels may be differentiated using Doppler imaging to detect pulsatile flow in the vessel (43). Arteries can be differentiated from veins due to their noncollapsible nature (37). After clipping and aseptic preparation of the desired site, the probe is generally oriented either transversely (short axis) or longitudinally (long axis) to the vessel, and the needle is advanced through the skin from several centimeters away from the probe $(21-23)$. The use of a real-time approach to advance the needle is preferred over using the US to locate a landmark prior to non-US-guided needle insertion. Evidence regarding superiority of one orientation of the probe in the human literature has been mixed (42, 44-48). Meta-analyses of probe orientation for multiple US-guided vascular access sites failed to find any significant difference for the first-pass success rate, mean time to success, mean attempts to success, and incidence of hematoma formation (49). Each view may have benefits, and developing familiarity with both is useful. The transverse view allowed for superior identification of other vessels but shows only a cross section of the desired vessel, making puncture more challenging. In contrast, a longitudinal view of the vessel allows for monitoring of the needle as it is advanced, though precise alignment of the plane of the probe and the course of the needle is needed, and visualization of other vessels or nerves may be lost. An oblique technique has also been described, which may combine the advantages of each view, but requires greater familiarity with US (42).

In human medicine, meta-analysis of US-guided catheter placement showed improved success of catheter placement, increased speed of placement, and decreased rate of complications as compared with blind or landmark-based placement, including in emergency patients $(27,29)$. The best evidence exists in aiding central venous catheterization, though benefit has also been shown with peripheral access $(22,41,50)$. US guidance is therefore increasingly recommended as the standard of care $(24,41-43,51,52)$. In veterinary medicine, US guidance is still being investigated. A study in healthy anesthetized canines did not find an improvement in the time to vascular access ( $45 \mathrm{~s}$ for US guidance vs. $7 \mathrm{~s}$ for landmark placed) or success (97 vs. 95\%) of central jugular catheter placement compared with a landmark-based technique (40). More applicably to critically ill patients, an experimental cardiopulmonary resuscitation (CPR) model in dogs demonstrated feasibility of ultrasound-guided jugular access with an average time to vascular access of 2-4 min (43). Further studies are needed to evaluate the feasibility and benefits of such a technique in critically ill dogs and cats.

\section{INTRAOSSEOUS ACCESS}

Because of the non-compressible nature of bone, intraosseous (IO) access offers a consistent route of access to the systemic circulation in the face of hypotension and hemodynamic collapse. While initially implemented largely in human pediatrics, use was expanded to adult patients (53). It is increasingly being advocated as a first-line option in both the prehospital and ER settings when rapid peripheral IV access is unsuccessful, including during CPR (53). Depending on patient size, access can be obtained using the stylet of an over-the-needle IV catheter or hypodermic needle (both reserved for young patients), using a manually placed bone marrow needle (e.g., Jamshidi), or using a purpose built IO catheter, often inserted via a bone injection gun or proprietary power driver $(24,27,54)$. The latter modality is a semiautomated device that has been shown to improve speed of placement (54-57). Limitations of placement include need for specialized equipment (except in case of using a hypodermic needle), which may restrict availability. Flow rate is also limited by needle diameter and the bone selected (58). Contraindications include osteomyelitis, regional pyoderma, preexisting fracture, and orthopedic hardware in the location of interest $(53,54)$. Possible complications reported in humans include osteomyelitis, fat embolism, fluid extravasation, nerve injury, compartment syndrome, and bone fractures. The overall rate of complications in humans has been reported at $<1 \%$ $(53,59,60)$, with the most common being extravasation of fluid, which can result in local tissue damage depending on the extravasated substance and potentially compartment syndrome $(53,61)$. Compartment syndrome has not been reported in dogs or cats with IO catheterization. However, careful monitoring for extravasation is imperative. Overall complication rates have not been documented in dogs and cats (54).

In dogs, successful placement has been described in the proximal humerus, lateral humeral condyle, trochanteric fossa, wing of the ilium, and medial proximal tibia $(27,54,58)$. A canine cadaveric study found the proximal humerus and distal femur to have the best combination of high flow rates and ease of access (58). IO access has also been investigated in cats at the medial tibia and proximal humerus (56). 
A Ultrasound Guided Catheter Placement

1. Clip and aseptically prepare the skin. For central catheter placement, sterile gloves are necessary

2. To maintain sterility, a sterile sheath can be placed over the US probe. A sterile glove containing ultrasound gel can be used as an alternative.

3. A high frequency $(5-15 \mathrm{MHz})$ linear probe is preferred, though for small patients a curvilinear probe may be used.

4. Using the US probe held in the non-dominant hand, localize the desired vein and assess along the vessel for anatomic variations or thrombosis.

a. Veins are differentiated from arteries due to their collapsible nature.

5. Either a short axis or long axis view of the desired vein can be obtained.

6. Insert the catheter needle at a 45 degree angle immediately adjacent to the US probe.

a. The needle tip should be visualized via US at all times when advancing.

b. The needle tip is advanced until puncture through the proximal vessel wall is visualized. The catheter is then advanced off of the stylet and into the vessel in routine fashion.

7. If placing a catheter using a guidewire, confirm the placement of the guidewire and subsequent catheter using US.

B Intraosseous Catheter Placement

1. The desired placement site should be clipped and aseptically prepared. Sterile gloves are recommended.

a. Sites for placement include the proximal humerus, trochanteric fossa, lateral humeral condyle, ilium, and medial proximal tibia.

2. Depending on the patients size and age, the appropriate needle size is selected.

a. In young animals, an 18-22 gauge hypodermic needle can be used. Smaller needles can be used in neonates.

b. In older animals, a bone marrow or commercial IO needle is necessary.

3. In conscious patients, local anesthesia (e.g. lidocaine) should be applied to the level of the periosteum.

4. A stab incision through the subcutaneous tissues over the desired placement site is made.

5. Manufacturer instruction should be followed depending on the type of needle utilized.

a. If using a power driver such as the $\mathrm{EZ}-\mathrm{IO}^{\mathrm{a}}$, an appropriate size needle is selected based on patient weight and bone. The needle and stylet are then attached to the drill, and force is applied at a perpendicular angle to the desired insertion surface to drill through the proximal cortex.

i. Suggested EZ-IO needle sizes: $<5 \mathrm{~kg}-15 \mathrm{~mm}$ needle; $5-39 \mathrm{~kg}-25 \mathrm{~mm}$ needle; $>39 \mathrm{~kg}$ : $45 \mathrm{~mm}$

6. Care should be taken to ensure only the proximal cortex is penetrated.

a. If the catheter tip is seated in the distal cortex, flow will likely be compromised and the catheter should be withdrawn slightly.

7. Once placed, the catheter should be firmly seated in the bone. Bone marrow aspiration via a syringe confirms placement, but may not occur in all successful placements. If placement is unsuccessful, a new bone should be selected to prevent extravasation.

8. A bolus of saline should flow easily, and the surrounding tissues be closely monitored for extravasation. This may also help to dislodge any marrow occluding the needle. Smaller needle sizes may encounter more resistance.

9. Slow infusion of lidocaine over 60 seconds through the catheter into the marrow cavity may decrease discomfort from initial fluid infusion.

10. The area should be bandaged and the catheter secured. Nursing care is then similar to a central IV catheters. The catheter should be removed as soon as alternate access has been obtained.

FIGURE 1 | Procedure for ultrasound-guided catheter placement (A) and intraosseous catheter placement (B) in dogs and cats in order to obtain emergency vascular access.

Placement in the pelvic limbs may be desirable in a CPR setting, where chest compression and airway manipulation complicate attempts to access the thoracic limbs, although the greater distance between this site and the heart is a consideration given poor circulation (54). This technique is described in detail in Figures 1B, 2. Time of placement in a human cadaver model was faster than peripheral venous cutdown (3.9 vs. $7.6 \mathrm{~min})$ (31). The technique appears to have a rapid learning curve, with reported success rates of
$87.5 \%$ in a canine cadaver study (62). In that study, time to IO placement was also faster than jugular cutdown ( 0.9 vs. $3.6 \mathrm{~min}$ ) (62). Infusion of medications into the bone marrow can be painful, as is removal of the catheter. Administration of hypertonic solutions via this route is controversial due to the potential for marrow and muscle necroses (63). However, most CPR medications, isotonic fluids, and blood products can be safely administered $(27,53,54,56,63,64)$. These are rapidly absorbed in the central circulation, with comparable 
A

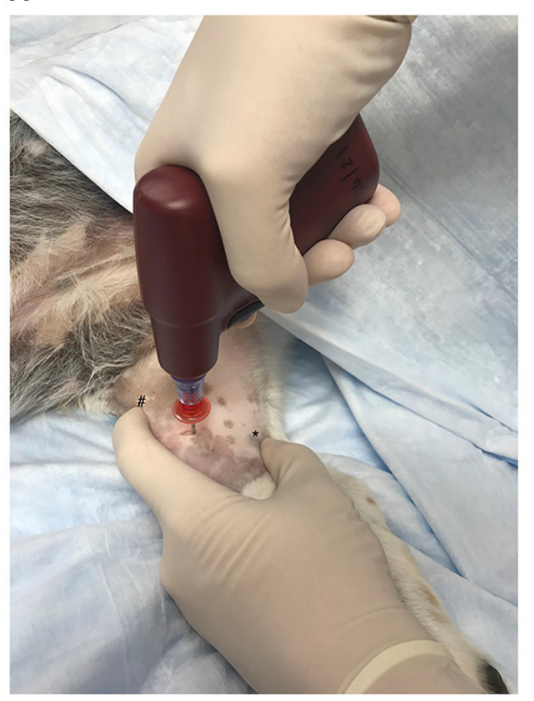

B

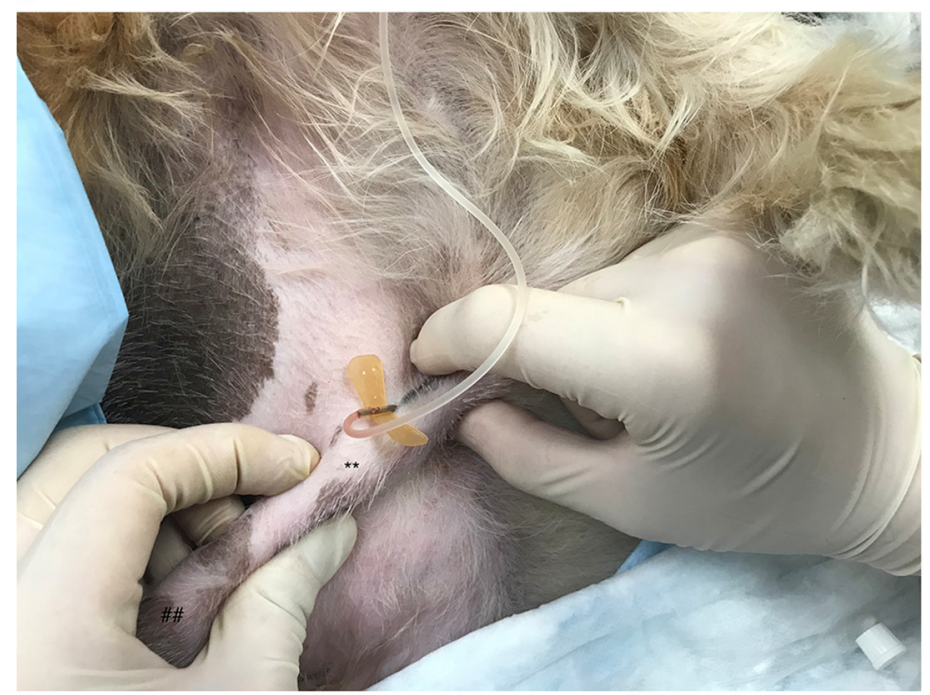

FIGURE 2 | (A) Emergency vascular access via intraosseous catheterization in the canine medial tibia using the EZ-IO G3 Power Driver (Vidacare Corp., Shavano Park, TX, USA). After aseptic preparation of the skin, a scalpel blade is used to make a small skin incision over the desired area. In a right-handed operator, the left index figure is used to palpate the patellar tendon (\#) to identify and avoid the stifle joint then placed directly distal to the joint on the cranial aspect of the tibia. The left thumb is then used to identify and secure the caudal aspect of the tibia $\left(^{*}\right)$. The remaining fingers of the left hand are used to stabilize the distal tibia. The G3 Power Driver is held in the right hand and positioned perpendicular to the tibia. The catheter is then advanced through the bone until a drop in resistance indicates the catheter has entered the medullary cavity. The catheter should be well-seated in the bone. Bone marrow aspiration via a syringe confirms placement. A bolus of saline should flow easily, and the surrounding tissues should be closely monitored for extravasation. (B) Placement of a catheter in the corpus cavernosum of a male canine. With the patient in lateral recumbency, the penis is isolated within the prepuce at the level of the caudal os penis (**). The pars longa glandis is on the left (\#\#). The needle is then inserted into the corpus cavernosum via the lateral aspect of the penis at an approximately $45^{\circ}$ angle, directed caudally. Aspiration of blood and easy flow of saline through the catheter confirm placement.

pharmacokinetics to IV administration (65). While IO catheters may remain in place for up to $72 \mathrm{~h}$, they should be removed as soon as another vascular access route has been established $(54,66,67)$.

Of additional interest, there has been recent research regarding the ability to derive point-of-care clinicopathologic information from marrow aspirated from the IO catheter at the time of placement. Studies in hemodynamically stable pigs, children, and adults have shown varying agreements between IO and venous samples for different analytes (57, 65, 68, 69). Only a few human studies focusing on hemodynamically unstable patients have been published to date. One group performed minimum database analysis measurements in hemodynamically unstable patients and found a clinically acceptable agreement for $\mathrm{pH}$, bicarbonate, base excess, and sodium and a moderate correlation for lactate and glucose, while $\mathrm{pCO}_{2}, \mathrm{pO}_{2}$, and potassium concentration did not show good agreement when IO and venous samples were compared (70). In another study, agreement declined after $15 \mathrm{~min}$ of CPR, particularly for acid base status (69). A recent veterinary study performed in healthy dogs anesthetized for orthopedic surgery demonstrated good agreement between IO aspirates and venous samples to assess minimum database variables [blood gas tensions, electrolytes, lactate, blood urea nitrogen (BUN), glucose, and packed cell volume/total protein (PCV/TP)], but not potassium or hematocrit (71). No veterinary paper has investigated the utility of IO samples in hemodynamically unstable dogs or cats. This would benefit from further study in critically ill patients.

\section{UMBILICAL VEIN}

In neonates, the umbilical vein (UV) provides an alternative route of access. The viability of the vessel depends on whether it has been previously ligated and is likely to be most accessible in the first day of life. This technique utilizes readily available material but can be more time-consuming and more difficult to perform than some alternatives if not routinely practiced and in the tight space constraints of a resuscitation setting (72). Contraindications include abnormal umbilical anatomy, omphalitis, and septic peritonitis $(73,74)$. Access is gained by first encircling the base of the umbilicus with umbilical tape and tightening to prevent hemorrhage. With the use of sterile technique, the umbilicus is then incised to expose the large, thin-walled vein, as well as the two smaller thick-walled umbilical arteries. A catheter is then advanced into the vein (73-75). It may be most practical to use the UV for single injections, which is 
readily achieved using a $25-\mathrm{G}$ needle. Care must be taken not to advance the catheter more than a few centimeters beyond the level at which a flash of blood is seen, as there is the potential for the catheter to lodge within the portal system if advanced too far into the central circulation without checking placement using a radiograph $(72,74,76)$. US-based placement confirmation has also been described in humans (77). Other complications include extravasation, thromboembolism, perforation of the peritoneum, and ischemia $(72,78)$.

In human neonates, $\mathrm{IO}$ is faster than UV catheterization in simulated resuscitation models, in both experienced and inexperienced hands (79-81). The average time required for IO catheter placement was $\sim 1-2 \mathrm{~min}$ faster than for UV catheter placement in simulated neonatal resuscitation (72, 79). Another study showed similar results and greater subjective ease of placement of the IO catheter in novice operators (80). Nevertheless, it is an important tool for vascular access in neonates when other routes are not available. Human studies comparing umbilical access with other routes in a clinical setting appear to be lacking, as are veterinary-specific studies.

\section{REFERENCES}

1. Hakim M, Shafy SZ, Uffman JC, Rice J, Raman VT, Tobias JD, et al. A survey to define and predict difficult vascular access in the pediatric perioperative population. Pediatric Health Med Ther. (2020) 11:27782. doi: 10.2147/PHMT.S260639

2. Walsh G. Difficult peripheral venous access: recognizing and managing the patient at risk. J Vasc Access. (2008) 13:198-203. doi: 10.2309/java.13-4-7

3. Petroski A, Frisch A, Joseph N, Carlson JN. Predictors of difficult pediatric intravenous access in a community emergency department. J Vasc Access. (2015) 16:521-6. doi: 10.5301/jva.5000411

4. Frey AM. Success rates for peripheral i.v. insertion in a children's hospital. Financial implications. J Intraven Nurs. (1998) 21:160-5.

5. Rauch D, Dowd D, Eldridge D, Mace S, Schears G, Yen K. Peripheral difficult venous access in children. Clin Pediatr. (2009) 48:895-901. doi: 10.1177/00099228093 35737

6. Yen K, Riegert A, Gorelick MH. Derivation of the DIVA score: a clinical prediction rule for the identification of children with difficult intravenous access. Pediatr Emerg Care. (2008) 24:143-7. doi: 10.1097/PEC.0b013e3181666f32

7. Loon FHJv, Puijn LAPM, Houterman S, Bouwman ARA. Development of the A-DIVA scale: a clinical predictive scale to identify difficult intravenous access in adult patients based on clinical observations. Medicine. (2016) 95:e3428-e. doi: 10.1097/MD.00000000000 03428

8. van Loon FHJ, van Hooff LWE, de Boer HD, Koopman S, Buise MP, Korsten HHM, et al. The modified A-DIVA scale as a predictive tool for prospective identification of adult patients at risk of a difficult intravenous access: a multicenter validation study. J Clin Med. (2019) 8:144. doi: $10.3390 / \mathrm{jcm} 8020144$

9. Juvin P, Blarel A, Bruno F, Desmonts JM. Is peripheral line placement more difficult in obese than in lean patients? Anesth Analg. (2003) 96:1218. doi: 10.1213/01.ANE.0000050570.85195.29

10. Dubick MA, Holcomb JB. A review of intraosseous vascular access: current status and military application. Mil Med. (2000) 165:552-9. doi: 10.1093/milmed/165.7.552

\section{CONCLUSION}

In conclusion, multiple options exist for patients with DVA. In particular, US-guided vascular access and IO catheters have become more readily available to practitioners, and both offer potential advantages. Further research is needed to verify their utility in unstable veterinary patients. Additionally, further research into DVA in veterinary patients could help to more rapidly identify patients that would benefit from these techniques.

\section{AUTHOR CONTRIBUTIONS}

JL, L-VG, GB, and CS contributed to the conception and design of the manuscript. JL wrote the first draft of the manuscript. All authors contributed to manuscript revision and read and approved the submitted version.

\section{FUNDING}

The funding for publication costs was provided by the University of Tennessee Veterinary Teaching Hospital Small Animal Clinical Sciences Department.

11. Rodriguez-Calero MA, de Pedro-Gomez JE, Molero-Ballester LJ, Fernandez Fernandez I, Matamalas-Massanet C, Moreno-Mejias L, et al. Risk factors for difficult peripheral intravenous cannulation. The PIVV2 multicentre casecontrol study. J Clin Med. (2020) 9:799. doi: 10.3390/jcm9030799

12. Shokoohi H, Loesche MA, Duggan NM, Liteplo AS, Huang C, Al Saud AA, et al. Difficult intravenous access as an independent predictor of delayed care and prolonged length of stay in the emergency department. JACEP Open. (2020) 1:1660-8. doi: 10.1002/emp2.12222

13. Seymour CW, Gesten F, Prescott HC, Friedrich ME, Iwashyna TJ, Phillips GS, et al. Time to treatment and mortality during mandated emergency care for sepsis. N Engl J Med. (2017) 376:2235-44. doi: 10.1056/NEJMoa1703058

14. Summers AM, Vezzi N, Gravelyn T, Culler C, Guillaumin J. Clinical features and outcome of septic shock in dogs: 37 cases (2008-2015). J Vet Emerg Crit Care. (2021) 31:360-70. doi: 10.1111/vec.13038

15. Lee SU, Jung JY, Ham EM, Wang SW, Park JW, Hwang S, et al. Factors associated with difficult intravenous access in the pediatric emergency department. J Vasc Access. (2020) 21:180-5. doi: 10.1177/1129729819865709

16. Song JH, Kim C, Yoo Y. Vein visualization using a smart phone with multispectral Wiener estimation for point-of-care applications. IEEE J Biomed Health Inform. (2015) 19:773-8. doi: 10.1109/JBHI.2014.2313145

17. Lewis W, Franco W. Smartphone imaging of subcutaneous veins. Lasers Surg Med. (2018) 50:1034-9. doi: 10.1002/lsm.22949

18. Blas J-VV, Carsten CG, III, Dooley J, Woo K, Fatula L, et al. Early validation of a tool to standardize vascular access procedure selection for hemodialysis. $J$ Vasc Surg. (2017) 65:11S-2S. doi: 10.1016/j.jvs.2017.03.024

19. van Oostrom H, Knowles TG. The clinical efficacy of EMLA cream for intravenous catheter placement in client-owned dogs. Vet Anaesth Analg. (2018) 45:604-8. doi: 10.1016/j.vaa.2018.03.009

20. Chebroux A, Leece EA, Brearley JC. Ease of intravenous catheterisation in dogs and cats: a comparative study of two peripheral catheters. J Small Anim Pract. (2015) 56:242-6. doi: 10.1111/jsap. 12318

21. Yasuda H, Yamamoto R, Hayashi Y, Kotani Y, Kishihara Y, Kondo N, et al. Occurrence and incidence rate of peripheral intravascular catheterrelated phlebitis and complications in critically ill patients: a prospective cohort study (AMOR-VENUS study). J Intensive Care Med. (2021) 9:3. doi: 10.1186/s40560-020-00518-4 
22. Pasalioglu KB, Kaya H. Catheter indwell time and phlebitis development during peripheral intravenous catheter administration. Pak J Med Sci. (2014) 30:725-30. doi: 10.12669/pjms.304.5067

23. Mandal A, Raghu K. Study on incidence of phlebitis following the use of pherpheral intravenous catheter. J Fam Med Prim Care. (2019) 8:282731. doi: $10.4103 /$ jfmpc.jfmpc_559_19

24. Hughes D, Beal MW. Emergency vascular access. Vet Clin N Am Small Anim Pract. (2000) 30:491-507. doi: 10.1016/S0195-5616(00)50036-9

25. Jayanthi NV, Dabke HV. The effect of IV cannula length on the rate of infusion. Injury. (2006) 37:41-5. doi: 10.1016/j.injury.2005.09.001

26. Davis H. Peripheral venous catheterization. In: Silverstein DC, Hopper K, editors. Small Animal Critical Care Medicine. 2nd ed. Missouri: Elsevier Saunders St Louis (2015). p. 1005-8.

27. Campbell MT, Macintire DK. Catheterization of the venous compartment. In: Burkitt Creedon JM, Davis H, editors. Advanced Monitoring and Procedures for Small Animal Emergency and Critical Care. Chichester: John WIley and sons (2012). p. 49-68.

28. McIntosh BB, Dulchavsky SA. Peripheral vascular cutdown. Crit Care Clin. (1992) 8:807-18. doi: 10.1016/S0749-0704(18)30226-4

29. Rhee KJ, Derlet RW, Beal SL. Rapid venous access using saphenous vein cutdown at the ankle. Am J Emerg Med. (1989) 7:263-6. doi: 10.1016/0735-6757(89)90166-6

30. Westfall MD, Price KR, Lambert M, Himmelman R, Kacey D, Dorevitch $\mathrm{S}$, et al. Intravenous access in the critically ill trauma patient: a multicentered, prospective, randomized trial of saphenous cutdown and percutaneous femoral access. Ann Emerg Med. (1994) 23:541-5. doi: 10.1016/S0196-0644(94)70074-5

31. Hubble MW, Trigg DC. Training prehospital personnel in saphenous vein cutdown and adult intraosseous access techniques. Prehosp Emerg Care. (2001) 5:181-9. doi: 10.1080/10903120190940100

32. Degim T, Dündaröz R, Sizlan A, Yaşar $M$, Denli $M$, Gökçay E. The use of the corpus cavernosum for the administration of phenobarbital: an experimental study in dogs. Int J Pharm. (2002) 246:105-9. doi: 10.1016/S0378-5173(02)00351-4

33. Nicol D, Watt A, Wood G, Wall D, Miller B. Corpus cavernosum as an alternative means of intravenous access in the emergency setting. Aust $\mathrm{N} \mathrm{Z}$ J Surg. (2000) 70:511-4. doi: 10.1046/j.1440-1622.2000.01859.x

34. Bradley M. Brief report: systemic vascular access and resuscitation via corpus cavernosum. Mil Med. (2016) 181:e14914. doi: 10.7205/MILMED-D-16-00001

35. Shafik A, El Sibai O, Shafik IA, Shafik AA. Corpora cavernosa as an alternative route for transfusion. Front Biosci. (2006) 11:2535-7. doi: 10.2741/1988

36. Stein M, Gray R. Corpus cavernosum as an emergency vascular access in dogs. Acad Radiol. (1995) 2:1073-7. doi: 10.1016/S1076-6332(05)80518-5

37. Blanco P. Ultrasound-guided peripheral venous cannulation in critically ill patients: a practical guideline. Ultrasound J. (2019) 11:27. doi: 10.1186/s13089-019-0144-5

38. Joing S, Strote S, Caroon L, Wall C, Hess J, Roline C, et al. Ultrasound-guided peripheral IV placement. N Engl J Med. (2012) 366:e38. doi: 10.1056/NEJMvcm1005951

39. Ortega R, Song M, Hansen CJ, Barash P. Ultrasound-guided internal jugular vein cannulation. $N$ Engl J Med. (2010) 362:e57. doi: 10.1056/NEJMvcm0810156

40. Hundley DM, Brooks AC, Thomovsky EJ, Johnson PA, Freeman LJ, Schafbuch $\mathrm{RM}$, et al. Comparison of ultrasound-guided and landmark-based techniques for central venous catheterization via the external jugular vein in healthy anesthetized dogs. Am J Vet Res. (2018) 79:628-36. doi: 10.2460/ajvr.79.6.628

41. Weiner MM, Geldard P, Mittnacht AJ. Ultrasound-guided vascular access: a comprehensive review. I Cardiothorac Vasc Anesth. (2013) 27:34560. doi: 10.1053/j.jvca.2012.07.007

42. Saugel B, Scheeren TWL, Teboul J-L. Ultrasound-guided central venous catheter placement: a structured review and recommendations for clinical practice. Crit Care. (2017) 21:225. doi: 10.1186/s13054-017-1814-y

43. Chamberlin SC, Sullivan LA, Morley PS, Boscan P. Evaluation of ultrasoundguided vascular access in dogs. J Vet Emerg Crit Care. (2013) 23:498503. doi: 10.1111/vec. 12102

44. Erickson CS, Liao MM, Haukoos JS, Douglass E, DiGeronimo M, Christensen E, et al. Ultrasound-guided small vessel cannulation: long-axis approach is equivalent to short-axis in novice sonographers experienced with landmark-based cannulation. West J Emerg Med. (2014) 15:82430. doi: 10.5811/westjem.2014.9.22404

45. Blaivas M, Brannam L, Fernandez E. Short-axis versus long-axis approaches for teaching ultrasound-guided vascular access on a new inanimate model. Acad Emerg Med. (2003) 10:1307-11. doi: 10.1197/S1069-6563(03)00534-7

46. Gottlieb M, Holladay D, Peksa GD. Comparison of short- vs long-axis technique for ultrasound-guided peripheral line placement: a systematic review and meta-analysis. Cureus. (2018) 10:e2718. doi: 10.7759/cureus.2718

47. Chen JY, Wang LK, Lin YT, Lan KM, Loh EW, Chen $\mathrm{CH}$, et al. Comparing short-, long-, and oblique-axis approaches to ultrasoundguided internal jugular venous catheterization: a meta-analysis of randomized controlled trials. J Trauma Acute Care Surg. (2019) 86:516-23. doi: 10.1097/TA.0000000000002158

48. Maitra S, Bhattacharjee S, Baidya DK. Comparison of long-, short-, and oblique-axis approaches for ultrasound-guided internal jugular vein cannulation: a network meta-analysis. J Vasc Access. (2020) 21:2049. doi: $10.1177 / 1129729819868927$

49. Gao Y-B, Yan J-H, Ma J-M, Liu X-N, Dong J-Y, Sun F, et al. Effects of long axis in-plane vs short axis out-of-plane techniques during ultrasound-guided vascular access. Am J Emerg Med. (2016) 34:77883. doi: 10.1016/j.ajem.2015.12.092

50. Stolz LA, Stolz U, Howe C, Farrell IJ, Adhikari S. Ultrasound-guided peripheral venous access: a meta-analysis and systematic review. J Vasc Access. (2015) 16:321-6. doi: 10.5301/jva.5000346

51. Butterfield M, Abdelghani R, Mohamad M, Limsuwat C, Kheir F. Using ultrasound-guided peripheral catheterization of the internal jugular vein in patients with difficult peripheral access. Am J Ther. (2017) 24:e6679. doi: 10.1097/MJT.0000000000000357

52. Milling TJ Jr., Rose J, Briggs WM, Birkhahn R, Gaeta TJ, et al. Randomized, controlled clinical trial of point-of-care limited ultrasonography assistance of central venous cannulation: the Third Sonography Outcomes Assessment Program (SOAP-3) Trial. Crit Care Med. (2005) 33:17649. doi: 10.1097/01.CCM.0000171533.92856.E5

53. Petitpas F, Guenezan J, Vendeuvre T, Scepi M, Oriot D, Mimoz O. Use of intra-osseous access in adults: a systematic review. Crit Care. (2016) 20:102. doi: 10.1186/s13054-016-1277-6

54. Giunti M, Otto CM. Intraosseous catheterization. In: Silverstein DC, Hopper K, editors. Small Animal Critical Care Medicine. 2nd ed. Missouri: Elsevier Saunders St Louis (2015). p. 1009-13.

55. Santos D, Carron PN, Yersin B, Pasquier M. EZ-IO(R) intraosseous device implementation in a pre-hospital emergency service: a prospective study and review of the literature. Resuscitation. (2013) 84:440-5. doi: 10.1016/j.resuscitation.2012.11.006

56. Bukoski A, Winter M, Bandt C, Wilson M, Shih A. Comparison of three intraosseous access techniques in cats. J Vet Emerg Crit Care. (2010) 20:3937. doi: 10.1111/j.1476-4431.2010.00558.x

57. Strandberg G, Eriksson M, Gustafsson MG, Lipcsey M, Larsson A. Analysis of intraosseous samples using point of care technology-an experimental study in the anaesthetised pig. Resuscitation. (2012) 83:13815. doi: 10.1016/j.resuscitation.2012.04.007

58. Lange J, Boysen SR, Bentley A, Atilla A. Intraosseous catheter flow rates and ease of placement at various sites in canine cadavers. Front Vet Sci. (2019) 6:312. doi: $10.3389 /$ fvets.2019.00312

59. Tyler JA, Perkins Z, De'Ath HD. Intraosseous access in the resuscitation of trauma patients: a literature review. Eur J Trauma Emerg Surg. (2020). 47:47-55. doi: 10.1007/s00068-020-01327-y

60. Suominen PK, Nurmi E, Lauerma K. Intraosseous access in neonates and infants: risk of severe complications - a case report. Acta Anaesthesiol Scand. (2015) 59:1389-93. doi: 10.1111/aas.12602

61. Atanda A Jr., Statter MB. Compartment syndrome of the leg after intraosseous infusion: guidelines for prevention, early detection, and treatment. Am J Orthop. (2008) 37:E198-200.

62. Allukian AR, Abelson AL, Babyak J, Rozanski EA. Comparison of time to obtain intraosseous versus jugular venous catheterization on canine cadavers. J Vet Emerg Crit Care. (2017) 27:506-11. doi: 10.1111/vec.12633

63. Alam HB, Punzalan CM, Koustova E, Bowyer MW, Rhee P. Hypertonic saline: intraosseous infusion causes myonecrosis in a dehydrated swine 
model of uncontrolled hemorrhagic shock. J Trauma. (2002) 52:1825. doi: 10.1097/00005373-200201000-00006

64. Hoskins SL, do Nascimento P, Jr., Lima RM, Espana-Tenorio JM, Kramer GC. Pharmacokinetics of intraosseous and central venous drug delivery during cardiopulmonary resuscitation. Resuscitation. (2012) 83:107-12. doi: 10.1016/j.resuscitation.2011. 07.041

65. Veldhoen ES, de Vooght KM, Slieker MG, Versluys AB, Turner NM. Analysis of blood gas, electrolytes and glucose from intraosseous samples using an i-STAT(囚) point-of-care analyser. Resuscitation. (2014) 85:35963. doi: 10.1016/j.resuscitation.2013.12.002

66. Philbeck TE, Puga TA, Montez DF, Davlantes C, DeNoia EP, Miller LJ. Intraosseous vascular access using the EZ-IO can be safely maintained in the adult proximal humerus and proximal tibia for up to $48 \mathrm{~h}$ : report of a clinical study. J Vasc Access. (2021). doi: 10.1177/1129729821992667. [Epub ahead of print].

67. Dev SP, Stefan RA, Saun T, Lee S. Insertion of an intraosseous needle in adults. N Engl J Med. (2014) 370:e35. doi: 10.1056/NEJMvcm1211371

68. Jousi M, Saikko S, Nurmi J. Intraosseous blood samples for point-of-care analysis: agreement between intraosseous and arterial analyses. Scand $J$ Trauma Resusc Emerg Med. (2017) 25:92. doi: 10.1186/s13049-017-0435-4

69. Abdelmoneim T, Kissoon N, Johnson L, Fiallos M, Murphy S. Acid-base status of blood from intraosseous and mixed venous sites during prolonged cardiopulmonary resuscitation and drug infusions. Crit Care Med. (1999) 27:1923-8. doi: 10.1097/00003246-199909000-00034

70. Tallman CI, Darracq M, Young M. Analysis of intraosseous blood samples using an EPOC point of care analyzer during resuscitation. Am J Emerg Med. (2017) 35:499-501. doi: 10.1016/j.ajem.2016.12.005

71. Ackert L, Boysen SR, Schiller T. A pilot study comparing bone marrow aspirates and venous blood for emergency point-of-care blood parameters in healthy dogs. J Vet Emerg Crit Care. (2019) 29:399-406. doi: 10.1111/vec.12858

72. Schwindt EM, Hoffmann F, Deindl P, Waldhoer TJ, Schwindt JC. Duration to establish an emergency vascular access and how to accelerate it: a simulationbased study performed in real-life neonatal resuscitation rooms. Pediatr Crit Care Med. (2018) 19:468-76. doi: 10.1097/PCC.0000000000001508

73. Anderson J, Leonard D, Braner DAV, Lai S, Tegtmeyer K. Umbilical vascular catheterization. N Engl J Med. (2008) 359:e18. doi: 10.1056/NEJMvcm08 00666

74. Tomek S, Asch S. Umbilical vein catheterization in the critical newborn: a review of anatomy and technique. EMS World. (2013) 42:50-2.
75. Lewis K, Spirnak PW. Umbilical Vein Catheterization. Treasure Island, FL: StatPearls Publishing (2021).

76. Traas AM. Resuscitation of canine and feline neonates. Theriogenology. (2008) 70:343-8. doi: 10.1016/j.theriogenology.2008.04.009

77. Meinen RD, Bauer AS, Devous K, Cowan E. Point-of-care ultrasound use in umbilical line placement: a review. J Perinatol. (2020) 40:5606. doi: 10.1038/s41372-019-0558-8

78. Selvam S, Humphrey T, Woodley H, English S, Kraft JK. Sonographic features of umbilical catheter-related complications. Pediatr Radiol. (2018) 48:196470. doi: 10.1007/s00247-018-4214-9

79. Rajani AK, Chitkara R, Oehlert J, Halamek LP. Comparison of umbilical venous and intraosseous access during simulated neonatal resuscitation. Pediatrics. (2011) 128:e954-8. doi: 10.1542/peds.20110657

80. Abe KK, Blum GT, Yamamoto LG. Intraosseous is faster and easier than umbilical venous catheterization in newborn emergency vascular access models. Am J Emerg Med. (2000) 18:126-9. doi: 10.1016/S0735-6757(00) 90001-9

81. Scrivens A, Reynolds PR, Emery FE, Roberts CT, Polglase GR, Hooper SB, et al. Use of intraosseous needles in neonates: a systematic review. Neonatology. (2019) 116:305-14. doi: 10.1159/0005 02212

Conflict of Interest: The authors declare that the research was conducted in the absence of any commercial or financial relationships that could be construed as a potential conflict of interest.

Publisher's Note: All claims expressed in this article are solely those of the authors and do not necessarily represent those of their affiliated organizations, or those of the publisher, the editors and the reviewers. Any product that may be evaluated in this article, or claim that may be made by its manufacturer, is not guaranteed or endorsed by the publisher.

Copyright $\odot 2021$ Lee, Guieu, Bussières and Smith. This is an open-access article distributed under the terms of the Creative Commons Attribution License (CC BY). The use, distribution or reproduction in other forums is permitted, provided the original author(s) and the copyright owner(s) are credited and that the original publication in this journal is cited, in accordance with accepted academic practice. No use, distribution or reproduction is permitted which does not comply with these terms. 